\title{
Therapeutic delivery of cyclin-A2 via recombinant adeno-associated virus serotype 9 restarts the myocardial cell cycle: An in vitro study
}

\author{
XIANG MA, AICHAO ZHAO, YONGZHAO YAO, WEN CAO, UJTT KARMACHARYA, \\ FEN LIU, BANGDANG CHEN, WANG BAOZHU, HUANG YING and YITONG MA \\ Department of Cardiovascular Medicine, First Affiliated Hospitalof Xinjiang Medical University, \\ Urumqi, Xinjiang 830054, P.R. China
}

Received December 30, 2013; Accepted September 18, 2014

DOI: $10.3892 / \mathrm{mmr} .2015 .3147$

\begin{abstract}
Cyclin-A2, which is downregulated following birth, has previously been established as a key regulator of the cell cycle. The present study aimed to detect the effects of cyclin-A2 on myocardial cells by using recombinant adeno-associated virus 9 (rAAV9). Sixty mice were selected and randomly divided into two groups $(n=30)$. The control group were injected with saline and the experimental group were transfected with the rAAV9-cyclinA2-CMV vector by intravenous injection into the tail vein. Tissues were harvested at two and four weeks following injection. Cyclin-A2 expression levels and localization were evaluated using western blot and immunohistochemical analyses. DNA synthesis and mitosis in the myocardium were confirmed by analyzing proliferating cell nuclear antigen (PCNA) and phospho-histone H3 (H3P) expression levels. Expression of Cyclin-A2 in the myocardium commenced two weeks following tail vein injection in the cyclin-A2-treated group, while no expression was observed in the control group. Four weeks following injection, expression levels of cyclin-A2 were higher than those observed at two weeks following injection into the myocardium (two weeks: $0.146 \pm 0.013$ vs. $27.1 \pm 3.33 \%, \mathrm{P}<0.001$; four weeks: $0.142 \pm 0.107$ vs. $74.4 \pm 3.36 \%, \mathrm{P}<0.001)$. PCNA displayed increased expression levels in the cyclin-A2-treated group (two weeks: $13.1 \pm 0.54$ vs. $65.8 \pm 3.44 \%, \mathrm{P}<0.001$; four weeks: $13.2 \pm 0.55$ vs
\end{abstract}

Correspondence to: Professor Yitong Ma or Professor Xiang Ma, Department of Cardiovascular Medicine, First Affiliated Hospital of Xinjiang Medical University, 137 LiYuShan South Road, Urumqi, Xinjiang 830054, P.R. China

E-mail: myt-xj@163.com

E-mail: maxiangxj@163.com

Abbreviations: PCNA, proliferating cell nuclear antigen; H3P, phospho-histone H3; rAAV9, recombinant adeno-associated virus serotype 9; CDK, cyclin-dependent kinase; eGFP, enhanced green fluorescent protein; CMV, cytomegalovirus

Key words: cyclin-A2, adeno-associated virus serotype 9, cell cycle
$71.2 \pm 1.58 \%, \mathrm{P}<0.001$ ); however, no change was observed in those of the control group. By contrast, no significant difference was observed in mitosis marker H3P expression levels between the two groups. Immunohistochemical analysis of cyclin-A2 indicated cytoplasmic, but not nuclear, localization. cyclin-A2 and PCNA expression levels in the liver, lung and kidney showed no significant difference between the two groups $(\mathrm{P}>0.05)$. It was therefore concluded that the delivery of cyclin-A2 via rAAV9 to the mouse myocardium restarted the myocardial cell cycle, thereby establishing steady and specific expression in the myocardium. Furthermore, the effect of Cyclin-A2 on the myocardium may provide a novel method for achieving cardiac regeneration following cardiac injury.

\section{Introduction}

Cyclin-A2 has an important role in the regulation of the cell cycle due to its two-point control. Cyclin-A2 interacts with CDK1 to control the $G_{1} / S$ transition and also interacts with CDK1 and CDK 2 to control the $G_{2} / M$ phase $(1,2)$. Cyclin-A2 has therefore previously been recognized as a key regulator of the cell cycle. Downregulation of cyclin A following birth has demonstrated time concordance with terminal cardiomyocyte cell-cycle exit in mammals (3). A recent study by Li et al (4) indicated that cyclin-A2 expression levels were increased following acute myocardial infarction (AMI), suggesting that cyclin-A2 may be involved in myocardial self repair. The study additionally reported that newborn cardiomyocytes were immature and that certain ventricular cardiomyocytes in rodents were multinucleated. Since this self repair following AMI was unable to prevent cardiac remodeling and the expression levels of cyclin-A2 were low (4), the present study hypothesized that overexpression of cyclin-A2 may promote the re-initiation of the cardiomyocyte cell cycle and cell division. However, overexpression of cyclin-A2 may result in excessive multiplication and potentially contribute to the development of cancer in other organs (1).

Adeno-associated virus (AAV) has previously been used as a gene therapy vector due to its low immunogenicity and sustained transgene expression (5). The inflammatory response caused by AAV is almost identical to that caused by saline or plasmids $(5,6)$. This feature makes AAV superior to 
other vectors, including adenovirus, herpes virus and lentivirus. For this reason, AAV vectors are able to provide safe, long-term gene transfer into several organs, including the lung (7), liver (8), brain (9), retina (10) and heart in animal models (11). Recently, AAV vectors exhibiting cardiac tropism facilitated cardiac transgene expression following intravenous injection (11). AAV serotype 9 (AAV9) has been proven to be a useful vector for gene therapy in cardiovascular disease via its specific transfection in the myocardium. Intravenous delivery or intrapericardial injection of AAV9-enhanced green fluorescence protein was demonstrated to produce higher gene expression levels in the myocardium than that of AAV5 and AAV6 (12-16). The recombinant AAV9 was therefore selected as a vector for cyclin-A2, driven by cytomegalovirus (CMV), for its superior cardiac tropism. The gene was transfected via tail vein injection and its efficiency was evaluated. The safety of the procedure was evaluated by detecting expression levels of cyclin-A2 and proliferation-associated proteins in the heart, lung, kidney and liver.

\section{Materials and methods}

Experimental animals. Male C57BL/6J mice (21-23 g), aged 10-12 weeks, were purchased from the animal center of Xinjiang Medical University (Urumqi, China). The mice were maintained at a temperature of $21-25^{\circ} \mathrm{C}$, in a light/dark cycle. Common feed was provided ad libitum. The present study was conducted in accordance with the recommendations of the Guide for the Care and Use of Laboratory Animals of the National Institutes of Health (Bethesda, MD, USA). The animal use protocol was reviewed and approved by the Institutional Animal Care and Use Committee of Xinjiang Medical University (Urumqui, China).

AAV9 recombinant ( $r A A V 9)$. The AAV9 vector (rAAV9-cyclinA2-CMV) was constructed by Virovek, Inc. (Hayward, CA, USA). The primers used were as follows: Forward, 5'-ATATGAATTCCACCAT GCCGGGCACCT CGAGGCA-3' and reverse, 5'-GGCCGTCGACTCACACA CTTAGTGTCTCTG-3'. The PCR reaction conditions were as follows: Initial denaturation at $95^{\circ} \mathrm{C}$ for $5 \mathrm{~min}, 35$ cycles of denaturation at $95^{\circ} \mathrm{C}$ for $30 \mathrm{sec}$, annealing at $56^{\circ} \mathrm{C}$ for $30 \mathrm{sec}$, extension at $72^{\circ} \mathrm{C}$ for $40 \mathrm{sec}$, and extension at $72^{\circ} \mathrm{C}$ for $5 \mathrm{~min}$. Following amplification by polymerase chain reaction, a final concentration of $2.39 \times 10^{13}$ genome copies (GC)/ml recombinant was obtained.

Experimental groups. Sixty C57BL/6J mice were randomly divided into control and experimental groups $(n=30$ per group). The experimental group were injected with $2 \times 10^{10} \mathrm{GC}$ rAAV9-cyclinA2-CMV recombinant in $200 \mu \mathrm{l}$ saline into the tail vein, while the control group were injected with the equivalent volume of saline. Observations were made at two and four weeks following injection.

Tissue samples. Two weeks following injection $(\mathrm{Tg}-2 \mathrm{w})$, the heart, liver, lung and kidney were harvested following sacrification of the animals by diastolic arrest induced by $0.2 \mathrm{~mol} / \mathrm{l}$ $\mathrm{KCl}$ (control group, $\mathrm{n}=7$; experimental group, $\mathrm{n}=8$ ). Remnant blood and fat tissue were removed from the myocardium and divided into two sections. One of these sections was immediately submerged in liquid nitrogen and the remaining section was fixed in paraformaldehyde or Bouin's fluid (HT10132; Sigma-Aldrich, St. Louis, MO, USA). Samples were stored at $-80^{\circ} \mathrm{C}$ until used. The liver, kidney and lungs were also stored. The procedure was repeated with the remaining mice at four weeks following injection ( $\mathrm{Tg}-4 \mathrm{w}$ ) (control group, $\mathrm{n}=5$; experimental group, $n=7$ ).

Western blot analysis. Tissue in 4-(2-hydroxyethyl)-1-piperazineethanesulfonic acid buffer with $0.5 \mathrm{mg} / \mathrm{ml}$ leupeptin, $10 \mu \mathrm{g} / \mathrm{ml}$ aprotinin and $1 \mathrm{mM}$ phenylmethanesulfonyl fluoride was homogenized in a grinder according to the bicinchoninic acid assay method (BCA Protein Assay kit; cat no. 23225; Pierce Biotechnology, Inc., Rockford, IL, USA). Firstly, diluted albumin (BSA) standards were prepared. Briefly, working reagents were prepared by mixing 50 parts of $\mathrm{BCA}^{\mathrm{TM}}$ Reagent A with 1 part of $\mathrm{BCA}^{\mathrm{TM}}$ Reagent B (50:1, Reagent A:B). A total of $0.1 \mathrm{ml}$ of each standard and samples were added to appropriately labeled test tubes, and $2 \mathrm{ml}$ working reagent was added to each at $37^{\circ} \mathrm{C}$. Subsequently, the absorbance of the samples was measured at $562 \mathrm{~nm}$ (SKanit for Multiskan GO 3.2; Thermo Fisher Scientific Inc., Rockford, IL, USA), in order to produce a standard curve. A standard curve was prepared by plotting the average blank-corrected $562 \mathrm{~nm}$ measurement for each BSA standard versus its concentration in $\mu \mathrm{g} / \mathrm{ml}$. Secondly, a microplate procedure was performed to determine the protein concentration. Briefly, $25 \mu \mathrm{l}$ of each standard or unknown sample was pipetted into a microplate well (working range $=20-2,000 \mu \mathrm{g} / \mathrm{ml}$ ). A total of $200 \mu \mathrm{l}$ of the working reagent was added to each well and the plate was mixed thoroughly on a plate shaker for $30 \mathrm{sec}$. The plate was then covered and incubated at $37^{\circ} \mathrm{C}$ for $30 \mathrm{~min}$. Subsequently, the plate was cooled to room temperature. The absorbance was measured at or near $562 \mathrm{~nm}$ on the microplate reader. The average $562 \mathrm{~nm}$ absorbance measurement of the blank standard replicates was subtracted from the $562 \mathrm{~nm}$ measurements of all other individual standard and unknown sample replicates. For electrophoresis, $50 \mu \mathrm{g}$ total protein from homogenized total tissue was purified by $12 \%$ SDS-PAGE (Invitrogen Life Technologies, Carlsbad, CA, USA) and subsequently blotted onto a $0.45 \mu \mathrm{m}$ polyvinylidene fluoride membrane. Mouse monoclonal immunoglobulin $\mathrm{G}_{1}\left(\mathrm{IgG}_{1}\right)$ anti-human cyclin-A2 (Santa Cruz Biotechnology, Inc., Dallas, TX, USA; 1:500; sc53227; $54 \mathrm{KDa}$ ), mouse monoclonal IgG2a anti-human proliferating cell nuclear antigen (PCNA; Cell Signaling Technology, Inc., Danvers, MA, USA; 1:1,000; 2586; 36 KDa) and rabbit polyclonal IgG anti-human phospho-histone $\mathrm{H} 3$ (H3P; Abcam, Cambridge, UK; 1:1,000; ab115152; 17 KDa) were used as the primary antibodies. Rabbit anti-human GAPDH (Cell Signaling Technology, Inc.; 1:1,000; 36 KDa) was used as reference. Following reaction with the primary antibodies at $4^{\circ} \mathrm{C}$ overnight, the membrane was washed with PBST and then incubated with secondary anti-rabbit (WP2007; Invitrogen Life Technologies) and anti-mouse IgG antibodies (WP2006; Invitrogen Life Technologies) for $2 \mathrm{~h}$ at room temperature. Then, the membranes were washed three times for 5 min and visualized on a gel imager (Gel Doc XR+; BioRad, CA, USA) to determine the optical density ratio with GAPDH. 
Table I. Expression levels of cyclin-A2, PCNA and H3P in the myocardium.

\begin{tabular}{lccccc}
\hline & \multicolumn{2}{c}{ Tg-2w } & & \multicolumn{2}{c}{ Tg-4w } \\
\cline { 2 - 3 } & Saline $(\mathrm{n}=5)$ & AAV9-cyclin-A2 $(\mathrm{n}=7)$ & & Saline ( $\mathrm{n}=5)$ & AAV9-cyclin-A2 $(\mathrm{n}=7)$ \\
\hline Cyclin-A2/GAPDH & $0.146 \pm 0.013$ & $27.1 \pm 3.33$ & & $0.142 \pm 0.107$ & $74.4 \pm 3.36$ \\
PCNA/GAPDH & $13.1 \pm 0.54$ & $65.8 \pm 3.44$ & & $13.2 \pm 0.55$ & $71.2 \pm 1.58$ \\
H3P/GAPDH & $11.6 \pm 0.63$ & $11.6 \pm 0.78$ & & $11.7 \pm 0.82$ & $11.6 \pm 0.78$ \\
\hline
\end{tabular}

PCNA, proliferating cell nuclear antigen; H3P, phospho-histone H3; Tg-2w, two weeks following injection; Tg-4w, four weeks following injection; AAV9, adeno-associated virus serotype 9. Values are presented as the mean expression level (\%) \pm standard deviation.

Table II. Expression of cyclin-A2 in liver, kidney and lung.

\begin{tabular}{lccccc}
\hline & \multicolumn{2}{c}{ Tg-2w } & & \multicolumn{2}{c}{ Tg-4w } \\
\cline { 2 - 3 } \cline { 5 - 6 } & Saline $(\mathrm{n}=5)$ & AAV9-cyclin-A2 $(\mathrm{n}=7)$ & & Saline $(\mathrm{n}=5)$ & AAV9-cyclin-A2 $(\mathrm{n}=7)$ \\
\hline Liver & $0.229 \pm 0.005$ & $0.217 \pm 0.028$ & & $0.231 \pm 0.031$ & $0.220 \pm 0.025$ \\
Kidney & $0.129 \pm 0.005$ & $0.125 \pm 0.008$ & & $0.127 \pm 0.003$ & $0.124 \pm 0.01$ \\
Lung & $11.6 \pm 0.63$ & $11.6 \pm 0.78$ & & $11.7 \pm 0.82$ & $11.6 \pm 0.78$ \\
\hline
\end{tabular}

PCNA, proliferating cell nuclear antigen; AAV9, adeno-associated virus serotype 9; Tg-2w, two weeks following injection; Tg-4w, four weeks following injection. Values are presented as the mean expression level $(\%) \pm$ standard deviation.

Immunohistochemistry. Tissues were sectioned into $5-\mu \mathrm{m}$ slices following Bouin's fixation. Paraffin-embedded samples were deparaffinized in xylol for 20 min followed by a descending series of ethanol (100, 95 and $70 \%)$ and distilled water. Subsequently, the sections were exposed to $3 \% \mathrm{H}_{2} \mathrm{O}_{2}$ for 20 min to block unspecific antigens, prior to being washed three times in phosphate-buffered saline (PBS) and incubated with sodium citrate for antigen retrieval at $92-98^{\circ} \mathrm{C}$ for $10 \mathrm{~min}$. Sections were washed three times in PBS following recovery at room temperature and goat serum was used to block unspecific antigens, following which the primary antibodies were added to the sections at the appropriate dilutions (cyclin-A2, 1:500; PCNA, 1:1,000; H3P, $1: 1,000)$.

Immunofluorescence. Immunofluorescence was used to detect the expression of cyclin-A2 in cardiomyocytes. Samples stored at $-80^{\circ} \mathrm{C}$ were gradually warmed to room temperature. Subsequently, the sections were washed three times in PBS for five minutes. Goat serum was added to block the unspecific antigen. The primary antibodies for cyclin-A2, PCNA and H3P diluted in 5\% bovine serum albumin were incubated with the tissue overnight at $4^{\circ} \mathrm{C}$. Corresponding secondary antibodies (goat anti-rabbit IgG and donkey anti-Mouse IgG CF ${ }^{\text {TM }}$ 594; Thermo Fisher Scientific Inc.) were incubated at $37^{\circ} \mathrm{C}$ for one hour and subsequently the nuclei were stained with DAPI for seven minutes at room temperature. Following three washes in PBS, images were captured of the stained sections using a Leica Photomicrograph (Leica Microsystems GmbH, Wetzlar, Germany).

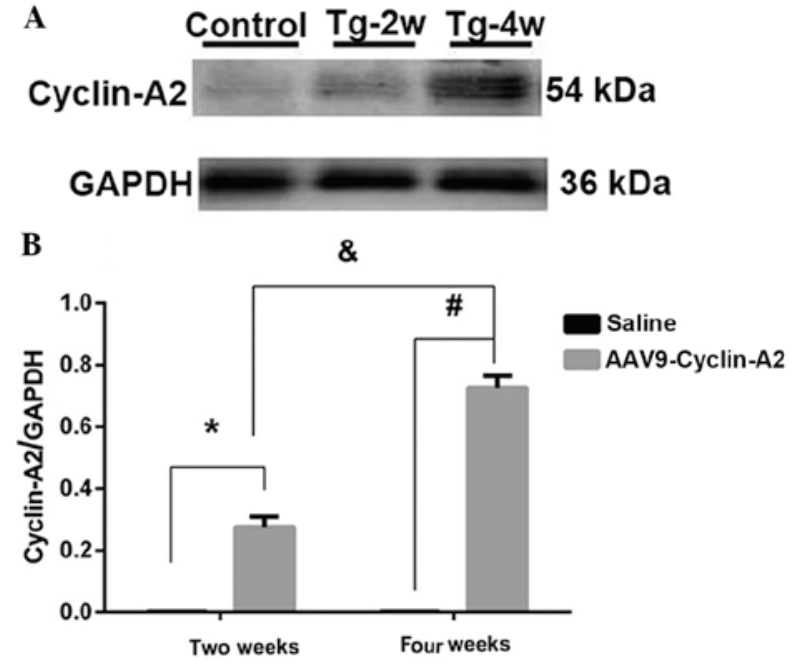

Figure 1. Expression of cyclin-A2 in the myocardium of the experimental and control groups. (A) Representative western blot analysis of cyclin-A2 expression in the myocardium of the control group and the experimental group at $\mathrm{Tg}-2 \mathrm{w}$ and $\mathrm{Tg}-4 \mathrm{w}$. Expression levels were normalized to GAPDH. (B) Cyclin-A2 expression levels were significantly higher in the AAV9-cyclin-A2 group than those of the control (saline) group and two and four weeks following injection. Values are expressed as the mean \pm standard deviation. Saline, $\mathrm{n}=5$ and AAV9-cyclin-A2, $\mathrm{n}=7$. ${ }^{*} \mathrm{P}<0.0001$ vs. saline group at two weeks. ${ }^{\#} \mathrm{P}<0.001$ vs. saline group at four weeks. Tg-2w, two weeks following injection; $\mathrm{Tg}-4 \mathrm{w}$, four weeks following injection; AAV9, adeno-associated virus serotype 9.

Statistical analysis. Values are expressed as the mean \pm standard deviation. Statistical significance between two groups was examined by Student's t-test and multigroup comparisons were 

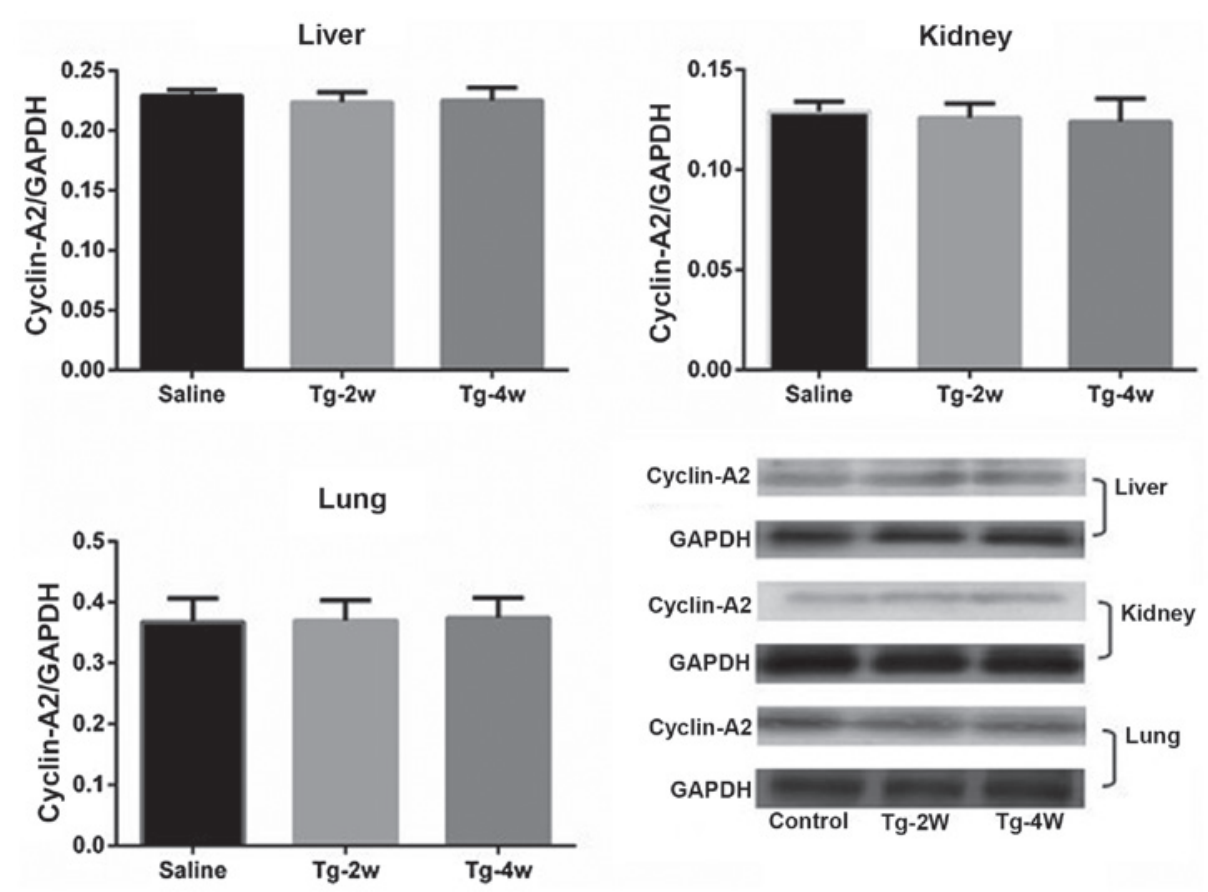

Figure 2. Western blot analysis was used to detect the expression of cyclin-A2 in the liver, kidney and lung at various time-points. Values are expressed as the mean \pm standard deviation. No significant difference was observed between cyclin-2A expression levels in the experimental and saline groups. P $>0.05$ in liver, kidney and lung. Saline group, $\mathrm{n}=5$. Cyclin-A2 group, $\mathrm{n}=7$. Tg-2w, two weeks following injection; Tg-4w, four weeks following injection.

made using one-way analysis of variance. Data were analyzed using SPSS 16.0 (SPSS, Inc., Chicago, IL, USA). P<0.05 was considered to indicate a statistically significant difference between values.

\section{Results}

Expression and location of cyclin-A2 in the myocardium. Western blot analysis indicated that expression of cyclin-A2 commenced two weeks $(27.1 \pm 3.33 \%)$ following injection and expression levels had increased four weeks following injection $(74.4 \pm 3.36 \%)$ in comparison to those at two weeks. Significantly lower expression levels were observed in the control group $(\mathrm{P}<0.0001 ; 0.146 \pm 0.013$ and $0.142 \pm 0.107 \%$, respectively; Fig. 1A and B; Table I). No significant difference was observed in expression levels between the two groups in the liver, kidney or lung (Fig. 2, Table II). The localization of cyclin-A2 expression was detected by immunohistochemical and immunofluorescent analysis, which indicated cytoplasmic, but not nucleic, expression (Figs. 3 and 4).

Influence of transfection on cardiomyocyte regeneration. Expression levels of PCNA were significantly higher in the cyclin-A2-treated group compared with those of the control group (two weeks: $13.1 \pm 0.54$ vs. $65.8 \pm 3.44, \mathrm{P}<0.001$; four weeks: $13.2 \pm 0.55$ vs. $71.2 \pm 1.58$; $\mathrm{P}<0.0001$; Fig. $5 \mathrm{~A}-\mathrm{C}$, Table I). In the cyclin-A2-treated group, no significant difference in cyclin-A2 expression was observed between two and four weeks following transfection, which revealed stable expression of cyclin-A2. This may indicate that persistent expression of cyclin-A2 promoted cell cycle progression. Immunohistochemical analysis indicated higher expression levels of PCNA in the $\mathrm{Tg}-2 \mathrm{w}$ and $\mathrm{Tg}-4 \mathrm{w}$ groups compared with those of the control group. However, no significant difference was identified in the expression levels of mitosis-specific protein, H3P, between the cyclin-A2-treated and control groups (two weeks: $11.6 \pm 0.63$ vs. $11.6 \pm 0.78 \%$, $\mathrm{P}>0.05$; four weeks: $11.7 \pm 0.82$ vs. $11.6 \pm 0.78 \%, \mathrm{P}>0.05$; Fig. 5, Table I).

Evaluation of safety. Expression of PCNA in the liver, kidney and lung was used to evaluate the safety of cyclin-A2 gene transfer. Western blot analysis indicated no statistically significant difference in PCNA expression levels in the liver, kidney or lung in the cyclin-A2-treated group compared to those of the control group, which confirmed the safety of gene transfer (Fig. 6).

\section{Discussion}

The present study aimed to evaluate the effect of cyclin-A2 transfection into the myocardium via rAAV9. The associated safety issues were also assessed. Studies previously indicated that delivery of cyclin-A2 via adenovirus or transgenesis restarted the myocardial cell cycle and enhanced cardiac regeneration following myocardial infarction $(17,18)$. Another study demonstrated that cyclin-A2 expression levels peaked at two weeks following transfection and subsequently gradually decreased so that by the fourth week, expression levels had almost disappeared. This confirmed that gene transfer of cyclin-A2 via adenovirus did not result in long term expression (17). A further study revealed long term expression of cyclin-A2 in myocardial regeneration following myocardial infarction (18). However, these studies did not investigate the safety of cyclin-A2 gene transfer or whether the adenovirus vector may provide unsustained cyclin-A2 expression. A 

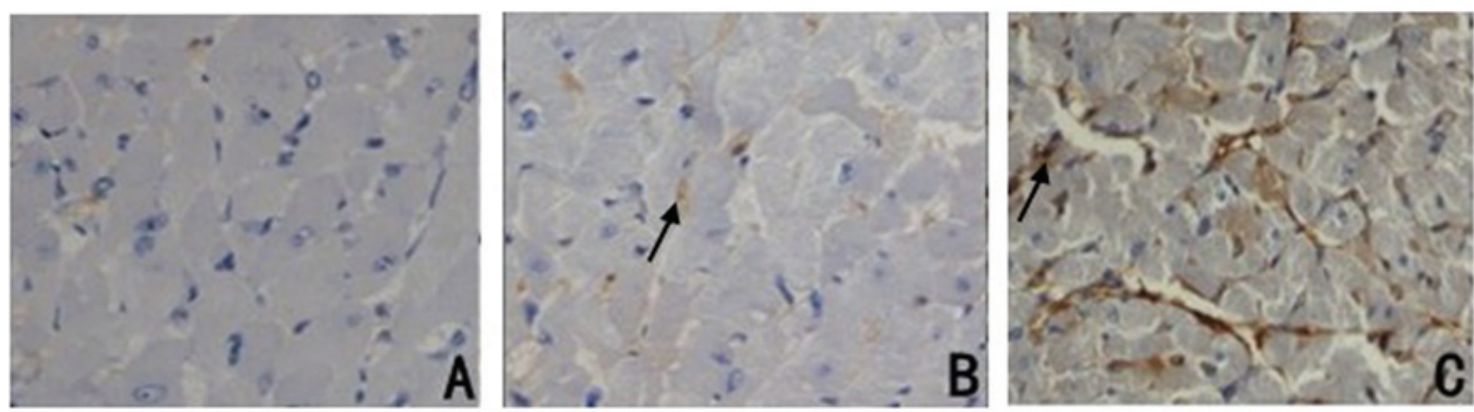

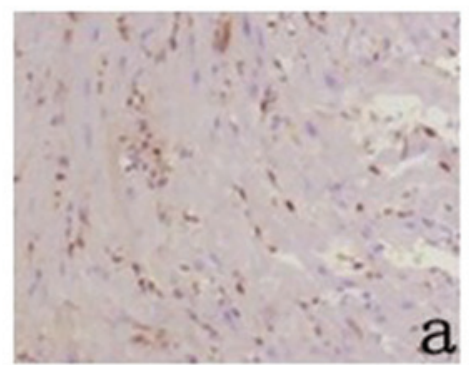

Control

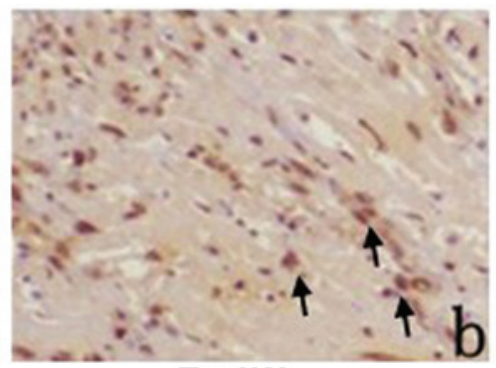

$\operatorname{Tg}-2 \mathrm{~W}$

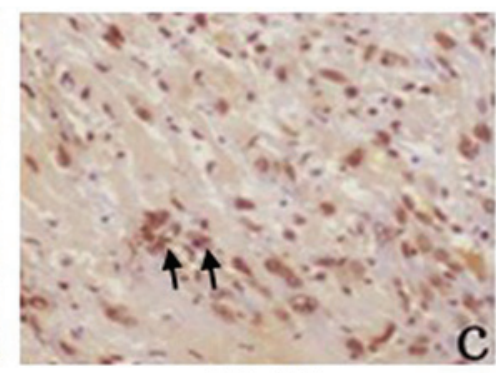

$\operatorname{Tg}-4 \mathrm{~W}$

Figure 3. Immunohistochemical analysis of cyclin-A2 and PCNA expression levels in the two groups. (A) Control group (saline). No cyclin-A2 expression was detected. (B) Tg-2w experimental group; arrows indicate positive cyclin-A2 expression in cardiomyocyte cytoplasm but not nucleus. (C) Tg-4w experimental group; arrows indicate cyclin-A2 positive expression in cardiomyocyte cytoplasm but not nucleus. Expression levels were significantly higher than those in the control and $\mathrm{Tg}-2 \mathrm{w}$ groups $(\mathrm{P}<0.0001)$. (a) PCNA expression localization in control group. (b) Tg- $2 \mathrm{w}$ expression of PCNA in cardiomyocytes of experimental group. Arrows indicate positive PCNA expression in cardiomyocyte nuclei. PCNA expression levels were significantly higher than those in the control group $(\mathrm{P}<0.001)$. (c) Tg-4w expression of PCNA in cardiomyocytes of experimental group. Arrows indicate positive PCNA expression in cardiomyocyte nuclei. There was no significant difference in PCNA expression levels compared with those in the Tg- $2 \mathrm{~W}$ group. However, there was a statistically significant difference between PCNA expression in the Tg- $2 \mathrm{w}$ and $\mathrm{Tg}-4 \mathrm{w}$ groups compared with that in the control group ( $\mathrm{P}<0.0001)$. 3,3'-Diaminobenzidine was used for immunohistochemical analysis (magnification, $\mathrm{x} 200$ ). Tg-2w, two weeks following injection; $\mathrm{Tg}-4 \mathrm{w}$, four weeks following injection.
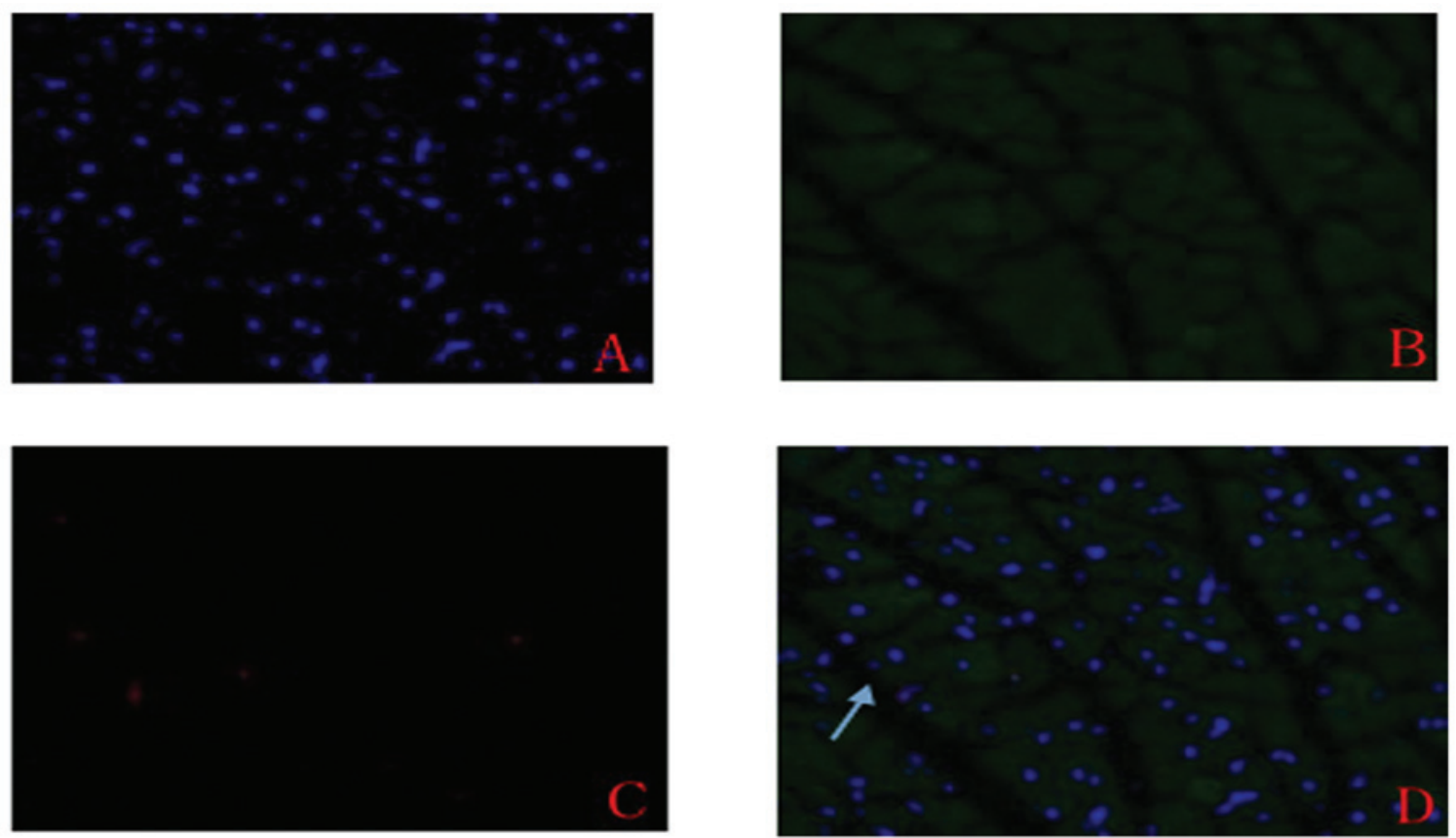

Figure 4. Immunofluorescent analysis of cyclin-A2 localization in the myocardium, (magnification, 200x). (A) DAPI staining of nuclei (blue). (B) Troponin I staining of the cytoplasm (green). (C) Cyclin-A2 (red). (D) Merge. Arrow indicates localization on cyclin-A2.

recent study discovered re-expression of cyclin-A2 following AMI, suggesting that cyclin-A2 may participate in myocardial self repair (4). Meanwhile, of cyclin-A2 also peaked at two weeks following AMI and levels were significantly decreased by four weeks, which was almost consistent with the observed effects of cyclin-A2 gene transfer (17). Therefore, to investigate the number of ways in which the exogenous cyclin-A2 gene performed, an rAAV9-cyclinA2-CMV complex was 

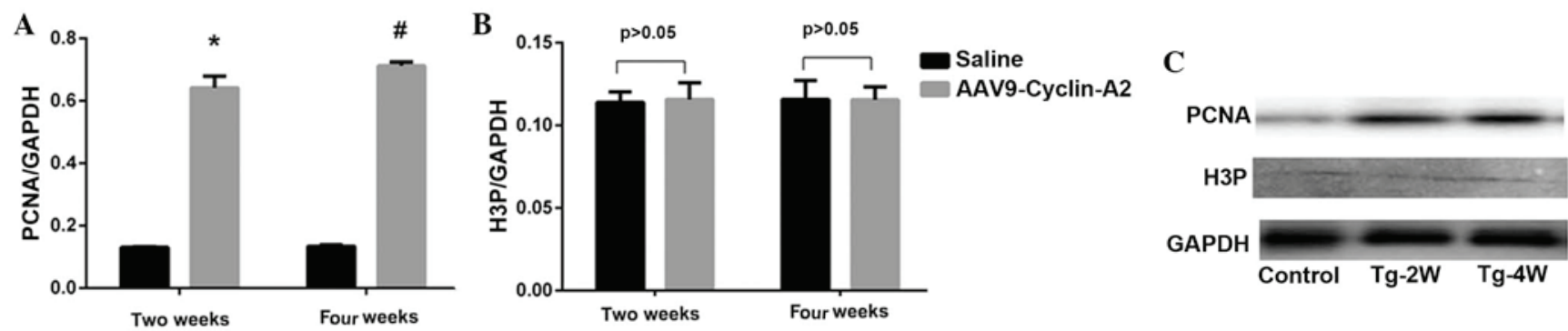

Figure 5. Expression levels of PCNA and H3P detected by western blot analysis. (A) PCNA expression levels displayed a significant difference between the control and experimental groups, two and four weeks following injection. ${ }^{*} \mathrm{P}<0.0001,{ }^{*} \mathrm{P}<0.0001$, AAV9-cyclin-A2 vs. saline group. (B) There was no significant difference in H3P expression levels between the two groups, $\mathrm{P}>0.05$. (control group, $\mathrm{n}=5$; experimental group, $\mathrm{n}=7$ ). Values are expressed as the mean \pm standard deviation. (C) Representative western blot of control group and experimental group at Tg-2w and Tg-4w. Size of proteins: PCNA, 36KD; H3P, $17 \mathrm{KDa}$; GAPDH, $36 \mathrm{KDa}$. Tg-2w, two weeks following injection; Tg-4w, four weeks following injection. PCNA, proliferating cell nuclear antigen; H3P, phospho-histone H3; AAV9, adeno-associated virus serotype 9.

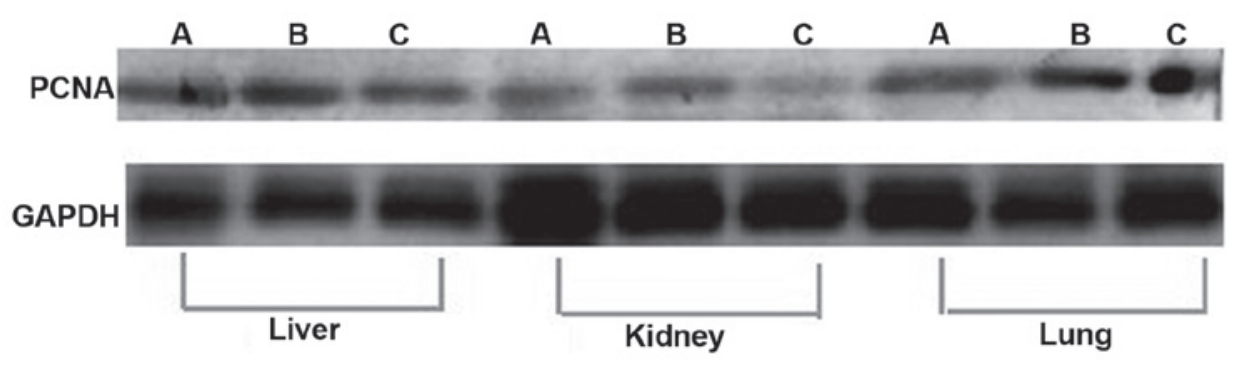

Figure 6. Expression of PCNA in liver, kidney and lung detected by western blot analysis. (A) Control group (saline). (B) Experimental group AAV9-cyclin-A2 at $\mathrm{Tg}-2 \mathrm{w}$. (C) Experimental group AAV9-cyclin-A2 at Tg-4w. There was no significant difference in expression levels between the control and experimental groups ( $\mathrm{P}>0.05)$. Control group, $\mathrm{n}=5 ; \mathrm{Tg}-2 \mathrm{w}, \mathrm{n}=7 ; \mathrm{Tg}-4 \mathrm{w}, \mathrm{n}=7$. Tg-2w, two weeks following injection; Tg-4w, four weeks following injection. PCNA, proliferating cell nuclear antigen.

constructed and injected into the myocardium of normal $\mathrm{C} 57 \mathrm{BL} / 6 \mathrm{~J}$ mice via the tail vein, in order to observe the expression levels and effects on the myocardial cell cycle following gene transfer.

Cyclin-A2 expression was observed two weeks following gene transfection and persisted for at least four weeks, whereas no expression was observed in the control group. The expression likely began at two weeks following transfection, as the single-stranded DNA of the AAV must be converted into double-stranded DNA prior to transcription (14). This process occurs rapidly in actively dividing cells, due to the presence of DNA polymerases (15). However, in post-mitotic cells and in particular, cardiomyocytes, the process is significantly delayed. The results of the present study are consistent with Svensson et al's (19) study on mice, which indicated that expression of cyclin-A2 may be sustained for a minimum of one month following rAAV transfection. rAAV is superior to the adenovirus in terms of expression duration, which was confirmed by the detection of lasting cyclin-A2 expression via rAAV9 transfer. It has been confirmed that an intermediate dose of AAV9 $\left(2.5 \times 10^{10} \mathrm{GC}\right)$ provides high-level gene transfer to the heart, whereas transfer is less via alternative AAV serotypes (16). Furthermore, at an intermediate dose, AAV9 expression is limited almost exclusively to the heart, with only a small number of positive cells detectable in the liver (12). The results of the present study demonstrated that expression levels of cyclin-A2 in the liver, lung and kidney showed no significant difference at two or four weeks following transfection compared with those of the control group. This therefore confirmed that AAV9 was the most suitable cardiotropic AAV serotype for gene transfer to the myocardium.

Following gene transfer, proliferation-associated proteins were also detected to evaluate the safety and efficiency of gene transfer. Higher expression of PCNA, a typical indicator of DNA synthesis, was observed in the cyclin-A2-treated group compared with that in the control group following gene transfer. This demonstrated that transfection with cyclin-A2 restarted the myocardial cell cycle and promoted DNA synthesis. Furthermore, no significant difference was observed in expression levels of PCNA between two and four weeks following transfection, indicating that exogenous cyclin-A2 was regulated by cell cycle-associated proteins. However, H3P, a mitosis-specific protein, exhibited no significant difference between the cyclin-A2-transfected and control groups; this was attributed to the cytoplasmic localization of cyclin-A2 following gene transfer. It has been confirmed that cyclin-A2 is localized predominantly in the nucleus during the $S$ phase; at the end of $G_{2}$ phase, it is re-localized to the centrosomes in the cytoplasm, where it binds to the poles of mitotic spindles (2). To facilitate its association with CDK2, cyclin-A2 is shuttled between the nucleus and cytoplasm and the nucleic localization of cyclin-A2 is required for mitosis $(20,21)$. However, cyclin-A2 expression via rAAV9 driven by CMV resulted in cytoplasmic localization, which may explain why no increase in cardiomyocyte mitosis was detected. 
Following gene transfer, cyclin-A2 expression levels in the liver, lung and kidney demonstrated no significant difference to those of the control group. This conclusion was confirmed by evaluating the expression levels of proliferation-associated proteins, PCNA and H3P, in the liver, lung and kidney. No significant difference was detected in PCNA or H3P expression levels between the liver, lung and kidney of the cyclin-A2-transfected group and those of the control group. This may reflect cardiac tropism from an alternative perspective but confirmed the safety of gene transfer.

In the present study, the safety and efficiency of gene transfer by rAAV9 was only confirmed in normal mice; further study should evaluate the effect of cyclin-A2 on the infarcted myocardium. Previous studies indicated that expression of genes transfected by adenovirus only lasted for four weeks; therefore, four weeks was selected as the experimental end-point. Further study should be conducted with an extended observation period, which may establish the extent of long-term gene expression following rAAV9 delivery. Further research is also required regarding myocardial regeneration.

In conclusion, the present study confirmed that the delivery of cyclin-A2 via an rAAV9 vector restarted the myocardial cell cycle and resulted in steady and specific cyclin-A 2 expression in the myocardium. This may provide a novel therapeutic route for myocardial regeneration following cardiac injury.

\section{References}

1. Yam CH, Fung TK and Poon RY: Cyclin A in cell cycle control and cancer. Cell Mol Life Sci 59: 1317-1326, 2002.

2. Pagano M1, Pepperkok R, Verde F, Ansorge W and Draetta G: Cyclin $\mathrm{A}$ is required at two points in the human cell cycle. EMBO J 11: 961-971, 1992.

3. Yoshizumi M, Lee WS, Hsieh CM, Tsai JC, Li J, Perrella MA, Patterson C, Endege WO, Schlegel R and Lee ME: Disappearance of cyclin A correlates with permanent withdrawal of cardiomyocytes from the cell cycle in human and rat hearts. J Clin Invest 95: 2275-2280, 1995.

4. Li Y, Hu S, Ma G, Yao Y, Yan G, Chen J, Li Y and Zhang Z: Acute myocardial infarction induced functional cardiomyocytes to re-enter cell cycle. Am J Transl Res 5: 327-335, 2013.

5. Mingozzi $F$ and High KA: Therapeutic in vivo gene transfer for genetic disease using AAV: progress and challenges. Nat Rev Genet 12: 341-355, 2011.

6. Wright MJ, Wightman LM, Lilley C, de Alwis M, Hart SL, Miller A, Coffin RS, Thrasher A, Latchman DS and Marber MS: In vivo myocardial gene transfer: optimization, evaluation and direct comparison of gene transfer vectors. Basic Res Cardiol 96 : 227-236, 2001
7. Flotte TR: Recent developments in recombinant AAV-mediated gene therapy for lung diseases (Review). Curr Gene Ther 5: 361-366, 2005.

8. Sands MS: AAV-mediated liver-directed gene therapy. Methods Mol Biol 807: 141-157, 2011.

9. Mandel RJ: CERE-110, an adeno-associated virus-based gene delivery vector expressing human nerve growth factor for the treatment of Alzheimer's disease. Curr Opin Mol Ther 12: 240-247, 2010.

10. Rolling F: Recombinant AAV-mediated gene transfer to the retina: gene therapy perspectives (Review). Gene Ther 11 (Suppl 1): S26-S32, 2011.

11. Pacak CA and Byrne BJ: AAV vectors for cardiac gene transfer: experimental tools and clinical opportunities (Review). Mol Ther 19: 1582-1590, 2011.

12. Bish LT, Morine K, Sleeper MM, Sanmiguel J, Wu D, Gao G, Wilson JM and Sweeney HL: Adeno-associated virus (AAV) serotype 9 provides global cardiac gene transfer superior to AAV1, AAV6, AAV7, and AAV8 in the mouse and rat. Hum Gene Ther 19: 1359-1368, 2008.

13. Bostick B, Ghosh A, Yue Y, Long C and Duan D: Systemic AAV-9 transduction in mice is influenced by animal age but not by the route of administration. Gene Ther 14: 1605-1609, 2007.

14. Zincarelli C, Soltys S, Rengo G and Rabinowitz JE: Analysis of AAV serotypes 1-9 mediated gene expression and tropism in mice after systemic injection. Mol Ther 16: 1073-1080, 2008.

15. Fang H, Lai NC, Gao MH, Miyanohara A, Roth DM, Tang T and Hammond HK: Comparison of adeno-associated virus serotypes and delivery methods for cardiac gene transfer. Hum Gene Ther Methods 23: 234-241, 2012.

16. Inagaki K, Fuess S, Storm TA, Gibson GA, Mctiernan CF, Kay MA and Nakai H: Robust systemic transduction with AAV9 vectors in mice: Efficient global cardiac gene transfer superior to that of AAV8. Mol Ther 14: 45-53, 2006.

17. Woo YJ, Panlilio CM, Cheng RK, Liao GP, Atluri P, Hsu VM, Cohen JE and Chaudhry HW: Therapeutic delivery of cyclin A2 induces myocardial regeneration and enhances cardiac function in ischemic heart failure. Circulation 114 (1 Suppl): I206-I213, 2006.

18. Cheng RK, Asai T, Tang H, Dashoush NH, Kara RJ, Costa KD, Naka Y, Wu EX, Wolgemuth DJ and Chaudhry HW: Cyclin A2 induces cardiac regeneration after myocardial infarction and prevents heart failure. Circ Res 100: 1741-1748, 2007.

19. Svensson EC, Marshall DJ, Woodard K, Lin H, Jiang F, Chu L and Leiden JM: Efficient and stable transduction of cardiomyocytes after intramyocardial injection or intracoronary perfusion with recombinat adeno-associated virus vectors. Circulation 99: 201-205, 1999.

20. Wang X, Song Y, Ren J and Qu X: Knocking-down Cyclin A(2) by siRNA suppresses apoptosis and switches differentiation pathways in K562 cells upon administration with doxorubicin. PLoS One 4: e6665, 2009.

21. Jackman M, Kubota Y, den Elzen N, Hagting A and Pines J: Cyclin A- and cyclin E-Cdk complexes shuttle between the nucleus and the cytoplasm. Mol Biol Cell 13: 1030-1045, 2002. 